\title{
Improvement in fast particle track reconstruction with robust statistics
}

M.G. Aartsen ${ }^{\text {b }}$, R. Abbasi ${ }^{\text {aa }}$, Y. Abdou ${ }^{\text {, }}$, M. Ackermann ${ }^{\text {ap }}$, J. Adams ${ }^{\circ}$, J.A. Aguilar u , M. Ahlers ${ }^{\text {aa }}$, D. Altmann ${ }^{\text {i }}$, J. Auffenberg aa ${ }^{\text {, X. Bai }}{ }^{\text {ae,1 }}{ }^{\text {, M. Baker }}{ }^{\text {aa }}$, S.W. Barwick ${ }^{\text {w }}$, V. Baum ${ }^{\text {ab }}$, R. Bay ${ }^{g}$, J.J. Beatty ${ }^{\text {q,r }}{ }^{\text {, S. Bechet }}{ }^{1}$, J. Becker Tjus ${ }^{j}$, K.-H. Becker ${ }^{\text {ao }}$, M.L. Benabderrahmane ${ }^{a p}$, S. BenZvi ${ }^{\text {aa }}$, P. Berghaus ${ }^{\text {ap }}$, D. Berley ${ }^{\mathrm{p}}$, E. Bernardini ${ }^{\text {ap }}$, A. Bernhard ${ }^{\text {ad }}$, D.Z. Besson ${ }^{y}$, G. Binder ${ }^{\text {g,h }}$, D. Bindig ao , M. Bissok a , E. Blaufuss ${ }^{\text {p }}$, J. Blumenthal $^{\text {a }}$, D.J. Boersma an , S. Bohaichuk ${ }^{t}$, C. Bohm ${ }^{\text {ah }}$, D. Bose ${ }^{\mathrm{m}}$, S. Böser ${ }^{\mathrm{k}}$, O. Botner ${ }^{\text {an }}$, L. Brayeur ${ }^{m}$, H.-P. Bretz ${ }^{\text {ap }}$, A.M. Brown ${ }^{\circ}$, R. Bruijn ${ }^{x}$, J. Brunner ap , M. Carson ${ }^{\mathrm{v}}$, J. Casey ${ }^{\mathrm{e}}$, M. Casier ${ }^{\mathrm{m}}$, D. Chirkin ${ }^{\text {aa }}$, A. Christov ${ }^{\mathrm{u}}$, B. Christy ${ }^{\mathrm{p}}$, K. Clark ${ }^{\text {am }}$, F. Clevermann ${ }^{\text {s }}$, S. Coenders ${ }^{a}$, S. Cohen ${ }^{x}$, D.F. Cowen ${ }^{a m, a l}$, A.H. Cruz Silva ap , M. Danninger ${ }^{\text {ah }}$, J. Daughhetee ${ }^{\mathrm{e}}$, J.C. Davis ${ }^{\mathrm{q}}$, M. Day ${ }^{\text {aa }}$, C. De Clercq $^{\mathrm{m}}{ }^{,}, \mathrm{S}$. De Ridder ${ }^{\mathrm{v}}$, P. Desiati ${ }^{\text {aa }}$, K.D. de Vries ${ }^{m}$, M. de With ${ }^{i}$, T. DeYoung am , J.C. Díaz-Vélez ${ }^{\text {aa }}$, M. Dunkman ${ }^{a m}$, R. Eagan ${ }^{a m}$, B. Eberhardt ${ }^{a b}$, J. Eisch ${ }^{\text {aa }}$, S. Euler ${ }^{a}$, P.A. Evenson ae ${ }^{\text {, }}$ O. Fadiran ${ }^{\text {aa }}$, A.R. Fazely ${ }^{\mathrm{f}}$, A. Fedynitch ${ }^{\mathrm{j}}$, J. Feintzeig ${ }^{\text {aa }}$, T. Feusels ${ }^{\mathrm{v}}$, K. Filimonov ${ }^{\mathrm{g}}$, C. Finley $^{\text {ah }}$, T. Fischer-Wasels ${ }^{\text {ao }}$, S. Flis ${ }^{\text {ah }}$, A. Franckowiak ${ }^{k}$, K. Frantzen ${ }^{s}$, T. Fuchs ${ }^{s}$, T.K. Gaisser ${ }^{\text {ae }}$, J. Gallagher ${ }^{\text {z }}$, L. Gerhardt ${ }^{\text {g,h }}$, L. Gladstone $^{\text {aa }}$, T. Glüsenkamp ${ }^{\text {ap }}$,

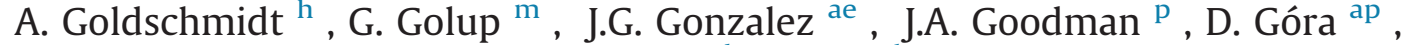
D.T. Grandmont ${ }^{\text {t }}$, D. Grant ${ }^{t}$, A. Groß ${ }^{\text {ad }}$, C. Ha ${ }^{\text {g,h }}$, A. Haj Ismail v , P. Hallen ${ }^{\text {a }}$, A. Hallgren an , F. Halzen ${ }^{\text {aa }}$, K. Hanson ${ }^{1}$, D. Heereman ${ }^{1}$, D. Heinen ${ }^{\text {a }}$, K. Helbing ao , R. Hellauer ${ }^{\mathrm{p}}$, S. Hickford ${ }^{\circ}$, G.C. Hill ${ }^{\mathrm{b}}$, K.D. Hoffman ${ }^{\mathrm{p}}$, R. Hoffmann ${ }^{\text {ao }}$, A. Homeier ${ }^{\mathrm{k}}$, K. Hoshina ${ }^{\text {aa }}$, W. Huelsnitz ${ }^{\text {p,2 }}$, P.O. Hulth ${ }^{\text {ah }}$, K. Hultqvist ${ }^{\text {ah }}$, S. Hussain ${ }^{\text {ae }}$, A. Ishihara ${ }^{\mathrm{n}}$, E. Jacobi ${ }^{\text {ap }}$, J. Jacobsen ${ }^{\text {aa }}$, K. Jagielski ${ }^{a}$, G.S. Japaridze ${ }^{d}$, K. Jero ${ }^{\text {aa }}$, O. Jlelati v ${ }^{\text {, }}$ B. Kaminsky ap , A. Kappes ${ }^{\text {i }}$, T. Karg ap , A. Karle aa , J.L. Kelley aa , J. Kiryluk ai , J. Kläs ${ }^{\text {ao }}$, S.R. Klein ${ }^{\text {g,h }}$, J.-H. Köhne ${ }^{\text {s }}$, G. Kohnen ${ }^{\text {ac }}$, H. Kolanoski ${ }^{\text {i }}$, L. Köpke ${ }^{\text {ab }}$, C. Kopper aa ${ }^{\text {, }}$ S. Kopper ao , D.J. Koskinen ${ }^{\text {am }}$, M. Kowalski ${ }^{k}$, M. Krasberg ${ }^{\text {aa }}$, K. Krings ${ }^{\text {a }}$, G. Kroll ${ }^{\text {ab }}$, J. Kunnen ${ }^{\mathrm{m}}$, N. Kurahashi ${ }^{\text {aa }}$, T. Kuwabara ${ }^{\text {ae }}$, M. Labare $^{\mathrm{v}}$, H. Landsman ${ }^{\text {aa }}$, M.J. Larson ${ }^{\text {ak }}$, M. Lesiak-Bzdak ${ }^{\text {ai }}$, M. Leuermann ${ }^{a}$, J. Leute ${ }^{\text {ad }}$, J. Lünemann ${ }^{\text {ab }}$, O. Macías ${ }^{\circ}$, J. Madsen ${ }^{\text {ag }}$, G. Maggi $^{\mathrm{m}}$, R. Maruyama ${ }^{\text {aa }}$, K. Mase $^{\mathrm{n}}{ }$, H.S. Matis ${ }^{\mathrm{h}}$, F. McNally ${ }^{\text {aa }}$, K. Meagher ${ }^{\mathrm{p}}$, M. Merck aa , T. Meures ${ }^{\text {l }}$, S. Miarecki ${ }^{\text {g,h }}{ }^{\text {, E. Middell ap }}{ }^{\text {ap N. Milke }}{ }^{\text {s }}$, J. Miller ${ }^{\mathrm{m}}$, L. Mohrmann ap , T. Montaruli ${ }^{\mathrm{u}, 3}$, R. Morse aa , R. Nahnhauer ${ }^{\text {ap }}$, U. Naumann ${ }^{\text {ao }}$, H. Niederhausen ${ }^{\text {ai }}$, S.C. Nowicki ${ }^{t}$, D.R. Nygren ${ }^{\mathrm{h}}$, A. Obertacke ${ }^{\text {ao }}$, S. Odrowski ${ }^{\mathrm{t}}$, A. Olivas ${ }^{\mathrm{p}}$, A. Omairat ${ }^{\text {ao }}$, A. O'Murchadha ${ }^{1}$, L. Paul ${ }^{\text {a }}$, J.A. Pepper ${ }^{\text {ak }}$, C. Pérez de los Heros ${ }^{\text {an }}$, C. Pfendner ${ }^{\mathrm{q}}$, D. Pieloth ${ }^{\mathrm{s}}$, E. Pinat ${ }^{\mathrm{l}}$, J. Posselt $^{\text {ao }}$, P.B. Price ${ }^{\mathrm{g}}$, G.T. Przybylski $^{\text {h }}$, L. Rädel $^{\text {a }}$, M. Rameez $^{\text {u }}$, K. Rawlins $^{\text {c }}$, P. Redl ${ }^{\text {p }}$, R. Reimann ${ }^{\text {a }}$, E. Resconi $^{\text {ad }}$, W. Rhode ${ }^{\mathrm{s}}$, M. Ribordy ${ }^{\mathrm{x}}$, M. Richman ${ }^{\mathrm{p}}$, B. Riedel aa ${ }^{\text {ad J.P. Rodrigues }}{ }^{\text {aa }}$, ${\text { C. } \text { Rott }^{\text {aj }} \text {, T. Ruhe }}^{\mathrm{s}}$, B. Ruzybayev ${ }^{\text {ae }}$, D. Ryckbosch ${ }^{\mathrm{v}}$, S.M. Saba ${ }^{\mathrm{j}}$, T. Salameh am H.-G. Sander ${ }^{a b}$, M. Santander ${ }^{\text {aa }}$, S. Sarkar af , K. Schatto ${ }^{\text {ab }}$, F. Scheriau ${ }^{\text {s }}$, T. Schmidt ${ }^{p}$, M. Schmitz ${ }^{\text {s }, ~ S . ~ S c h o e n e n ~}{ }^{\text {a }}$, S. Schöneberg ${ }^{j}$, A. Schönwald ${ }^{\text {ap }}$, A. Schukraft ${ }^{\text {a }}$, L. Schulte ${ }^{k}$, O. Schulz $^{\text {ad }}$, D. Seckel ae ${ }^{\text {, Y. Sestayo }}{ }^{\text {ad }}$, S. Seunarine ${ }^{\text {ag }}$, R. Shanidze ${ }^{\text {ap }}$, C. Sheremata ${ }^{t}$, M.W.E Smith am , D. Soldin ${ }^{\text {ao }}$, G.M. Spiczak ${ }^{\text {ag }}$, C. Spiering ap , M. Stamatikos ${ }^{\mathrm{q}, 4}$, 


\section{T. Stanev ae ${ }^{\text {, A. Stasik k }}{ }^{\text {, T. Stezelberger }}{ }^{\text {h }}$, R.G. Stokstad ${ }^{\text {h }}$, A. Stößl ap , E.A. Strahler ${ }^{m}$, R. Ström an , G.W. Sullivan ${ }^{\text {p }}$, H. Taavola ${ }^{\text {an }}$, I. Taboada ${ }^{\text {e }}$, A. Tamburro ae ${ }^{\text {a }}$, A. Tepe ${ }^{\text {ao }}$, S. Ter-Antonyan ${ }^{f}$, G. Tešić ${ }^{\text {am }}$, S. Tilav ${ }^{\text {ae }}$, P.A. Toale ${ }^{a k}$, S. Toscano ${ }^{\text {aa }}$, E. Unger ${ }^{j}$, M. Usner ${ }^{\mathrm{k}}$, S. Vallecorsa ${ }^{\mathrm{u}}$, N. van Eijndhoven ${ }^{\mathrm{m}}$, A. Van Overloop ${ }^{\mathrm{v}}$, J. van Santen aa ,

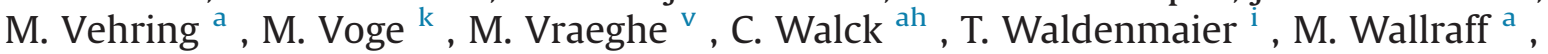 Ch. Weaver $^{\text {aa }}$, M. Wellons ${ }^{\text {aa,* }}$, C. Wendt ${ }^{\text {aa }}$, S. Westerhoff aa ${ }^{\text {a }}$ N. Whitehorn ${ }^{\text {aa }}$,

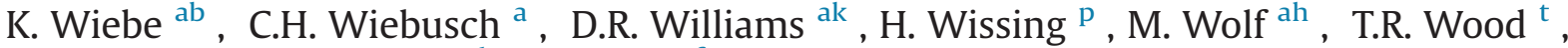 K. Woschnagg ${ }^{\mathrm{g}}$, D.L. Xu ${ }^{\text {ak }}$, X.W. Xu ${ }^{\mathrm{f}}$, J.P. Yanez ${ }^{\mathrm{ap}}$, G. Yodh ${ }^{\text {w }}$, S. Yoshida ${ }^{\mathrm{n}}$, P. Zarzhitsky ${ }^{\text {ak }}$, J. Ziemann ${ }^{\text {s }}$, S. Zierke ${ }^{a}$, M. Zoll ${ }^{\text {ah }}$, B. Recht ${ }^{\text {aq }}$, C. Ré ${ }^{\text {ar }}$}

a III. Physikalisches Institut, RWTH Aachen University, D-52056 Aachen, Germany

${ }^{\mathrm{b}}$ School of Chemistry \&' Physics, University of Adelaide, Adelaide 5005, SA, Australia

c Department of Physics and Astronomy, University of Alaska Anchorage, 3211 Providence Dr., Anchorage, AK 99508, USA

${ }^{\mathrm{d}}$ CTSPS, Clark-Atlanta University, Atlanta, GA 30314, USA

e School of Physics and Center for Relativistic Astrophysics, Georgia Institute of Technology, Atlanta, GA 30332, USA

f Department of Physics, Southern University, Baton Rouge, LA 70813, USA

${ }^{\mathrm{g}}$ Department of Physics, University of California, Berkeley, CA 94720, USA

${ }^{\mathrm{h}}$ Lawrence Berkeley National Laboratory, Berkeley, CA 94720, USA

i Institut für Physik, Humboldt-Universität zu Berlin, D-12489 Berlin, Germany

${ }^{\mathrm{j}}$ Fakultät für Physik \& Astronomie, Ruhr-Universität Bochum, D-44780 Bochum, Germany

${ }^{\mathrm{k}}$ Physikalisches Institut, Universität Bonn, Nussallee 12, D-53115 Bonn, Germany

${ }^{1}$ Université Libre de Bruxelles, Science Faculty CP230, B-1050 Brussels, Belgium

${ }^{\mathrm{m}}$ Vrije Universiteit Brussel, Dienst ELEM, B-1050 Brussels, Belgium

${ }^{\mathrm{n}}$ Department of Physics, Chiba University, Chiba 263-8522, Japan

${ }^{\circ}$ Department of Physics and Astronomy, University of Canterbury, Private Bag 4800, Christchurch, New Zealand

${ }^{\mathrm{P}}$ Department of Physics, University of Maryland, College Park, MD 20742, USA

${ }^{\mathrm{q}}$ Department of Physics and Center for Cosmology and Astro-Particle Physics, Ohio State University, Columbus, OH 43210, USA

${ }^{\mathrm{r}}$ Department of Astronomy, Ohio State University, Columbus, OH 43210, USA

${ }^{s}$ Department of Physics, TU Dortmund University, D-44221 Dortmund, Germany

${ }^{\mathrm{t}}$ Department of Physics, University of Alberta, Edmonton, Alberta, Canada T6G 2E1

u Département de physique nucléaire et corpusculaire, Université de Genève, CH-1211Genève, Switzerland

${ }^{v}$ Department of Physics and Astronomy, University of Gent, B-9000 Gent, Belgium

${ }^{\mathrm{w}}$ Department of Physics and Astronomy, University of California, Irvine, CA 92697, USA

${ }^{x}$ Laboratory for High Energy Physics, École Polytechnique Fédérale, CH-1015 Lausanne, Switzerland

${ }^{y}$ Department of Physics and Astronomy, University of Kansas, Lawrence, KS 66045, USA

${ }^{z}$ Department of Astronomy, University of Wisconsin, Madison, WI 53706, USA

aa Department of Physics and Wisconsin IceCube Particle Astrophysics Center, University of Wisconsin, Madison, WI 53706, USA

${ }^{\mathrm{ab}}$ Institute of Physics, University of Mainz, Staudinger Weg 7, D-55099 Mainz, Germany

ac Université de Mons, 7000 Mons, Belgium

${ }^{\text {ad }}$ T.U. Munich, D-85748 Garching, Germany

ae Bartol Research Institute and Department of Physics and Astronomy, University of Delaware, Newark, DE 19716, USA

af Department of Physics, University of Oxford, 1 Keble Road, Oxford OX1 3NP, UK

ag Department of Physics, University of Wisconsin, River Falls, WI 54022, USA

ah Oskar Klein Centre and Department of Physics, Stockholm University, SE-10691 Stockholm, Sweden

ai Department of Physics and Astronomy, Stony Brook University, Stony Brook, NY 11794-3800, USA

aj Department of Physics, Sungkyunkwan University, Suwon 440-746, Republic of Korea

${ }^{\text {ak }}$ Department of Physics and Astronomy, University of Alabama, Tuscaloosa, AL 35487, USA

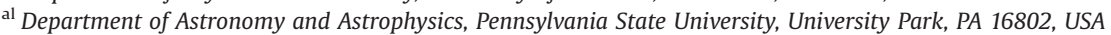

am Department of Physics, Pennsylvania State University, University Park, PA 16802, USA

an Department of Physics and Astronomy, Uppsala University, Box 516, S-75120 Uppsala, Sweden

ao Department of Physics, University of Wuppertal, D-42119 Wuppertal, Germany

ap DESY, D-15735 Zeuthen, Germany

${ }^{\text {aq }}$ Department of Computer Science, University of California, Berkeley, CA 94704, USA

ar Department of Computer Science, Stanford University, Stanford, CA 94305, USA

\section{A R T I C L E I N F O}

Article history:

Received 26 August 2013

Received in revised form

28 October 2013

Accepted 28 October 2013

Available online 6 November 2013

\section{A B S T R A C T}

The IceCube project has transformed $1 \mathrm{~km}^{3}$ of deep natural Antarctic ice into a Cherenkov detector. Muon neutrinos are detected and their direction is inferred by mapping the light produced by the secondary muon track inside the volume instrumented with photomultipliers. Reconstructing the muon track from the observed light is challenging due to noise, light scattering in the ice medium, and the possibility of simultaneously having multiple muons inside the detector, resulting from the large flux of cosmic ray muons.

\footnotetext{
* Corresponding author. Wisconsin Institutes for Discovery, 330 N. Orchard St., Madison, WI 53715, USA. Tel.: +1 3045424464.

E-mail addresses: wellons@icecube.wisc.edu, mwellons@gmail.com, mwellons@cs.wisc.edu (M. Wellons).
}

\footnotetext{
1 Physics Department, South Dakota School of Mines and Technology, Rapid City, SD 57701, USA

2 Los Alamos National Laboratory, Los Alamos, NM 87545, USA.

3 Sezione INFN, Dipartimento di Fisica, I-70126 Bari, Italy.

${ }^{4}$ NASA Goddard Space Flight Center, Greenbelt, MD 20771, USA
} 
Keywords:

IceCube

Track reconstruction

Neutrino telescope

Neutrino astrophysics

Robust statistics
This paper describes work on two problems: (1) the track reconstruction problem, in which, given a set of observations, the goal is to recover the track of a muon; and (2) the coincident event problem, which is to determine how many muons are active in the detector during a time window. Rather than solving these problems by developing more complex physical models that are applied at later stages of the analysis, our approach is to augment the detector's early reconstruction with data filters and robust statistical techniques. These can be implemented at the level of on-line reconstruction and, therefore, improve all subsequent reconstructions. Using the metric of median angular resolution, a standard metric for track reconstruction, we improve the accuracy in the initial reconstruction direction by $13 \%$. We also present improvements in measuring the number of muons in coincident events: we can accurately determine the number of muons $98 \%$ of the time.

\section{Introduction}

The IceCube neutrino detector searches for neutrinos that are generated by the universe's most violent astrophysical events: exploding stars, gamma ray bursts, and cataclysmic phenomena involving black holes and neutron stars [1]. The detector, roughly $1 \mathrm{~km}^{3}$ in size, is located near the geographic South Pole and is buried to a depth of about $2.5 \mathrm{~km}$ in the Antarctic ice [2]. The detector is illustrated in Fig. 1, and a more complete description is given in Section 2.

When a neutrino enters the telescope, it occasionally interacts in the ice and generates a muon. The neutrino direction can be inferred from a reconstruction of the muon track. Muons are also generated by cosmic rays interacting in the atmosphere, and separation of the background of cosmic ray muons and neutrinoinduced muons is a necessary step for neutrino analysis. This separation is challenging, as the number of observed cosmic ray muons exceeds the number of observed neutrino muons by over five orders of magnitude [3].

The primary mechanism for separating the cosmic ray muons from the neutrino muons is reconstructing the muon track and determining whether the muon was traveling downwards into the Earth or upwards out of the Earth. Because neutrinos can penetrate through the Earth but cosmic ray muons cannot, it follows that a muon traveling out of the Earth must have been generated by a neutrino. Thus, by selecting only the muons that are reconstructed as up-going, the cosmic ray muons can, in principle, be removed from the data. Because the number of cosmic ray

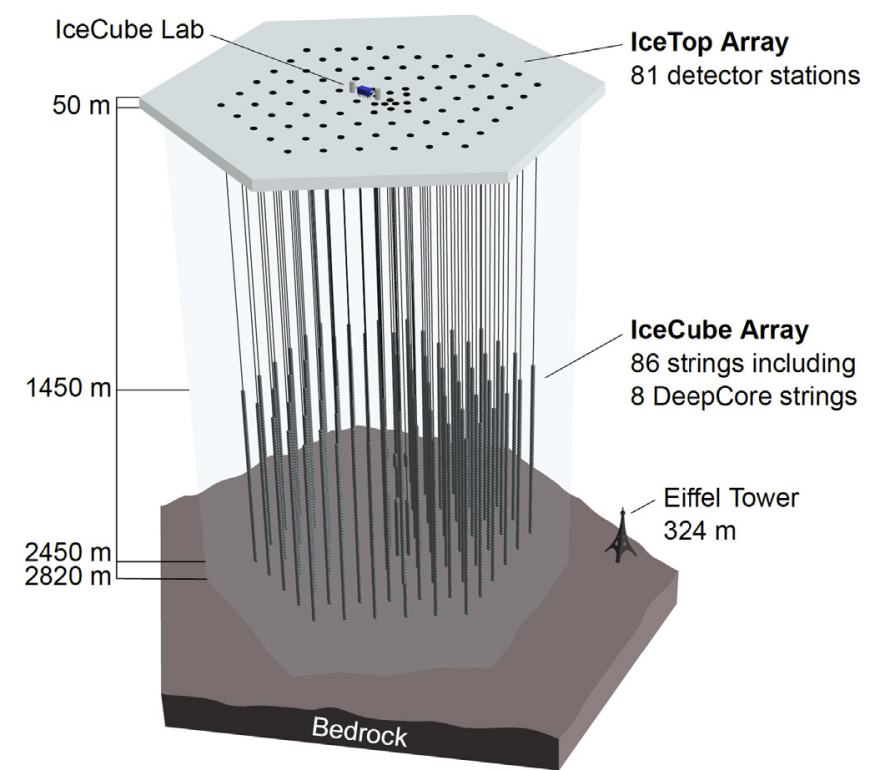

Fig. 1. The IceCube neutrino detector in the Antarctic ice. A picture of the Eiffe Tower is shown for scale. muons overwhelms the number of neutrino muons, high accuracy is critical for preventing erroneous reconstruction of cosmic ray muons as neutrino-induced.

Here, we examine two problems that arise in the separation of cosmic ray muons from neutrino muons in the IceCube detector:

1. Reconstruction, in which the track of a muon is reconstructed from the observed light at different positions and times in the detector.

2. Coincident event detection, in which we detect the number of muons inside the detector, and assign observed photons to a muon.

Sophisticated reconstruction techniques have been developed that computationally model in detail the muon's Cherenkov cone, as well as the scattering and absorption of photons through layers of Antarctic ice with varying optical properties [3-5]. Rather than further refining these techniques, the current work focusses on improving the statistical techniques and optimizing data filtering in the early online track reconstruction performed on the data in real time at the South Pole. Besides benefiting directly any analysis that uses the online reconstruction, such as the search for cosmogenic neutrinos, any later analysis will benefit from improvements made at the early stages of the data collection.

\subsection{Related work}

Track reconstruction and coincident event detection challenges are ubiquitous in particle physics [6-8], both in particle accelerators and cosmic particle detectors. While the work described in this paper builds on the previous technique developed for the IceCube detector [3], these techniques are general purpose and potentially have applications in detectors beyond IceCube.

\subsection{Outline}

We begin by describing the IceCube detector and track reconstruction challenges in Section 2. In Section 3, we describe the reconstruction pipeline including the prior IceCube software, then we present improvements to the online tracking algorithm and discuss the results. Section 4 describes the improvements on coincident event detection and follows a parallel structure to Section 3. We conclude in Section 5.

\section{IceCube detector and track reconstruction challenges}

The IceCube detector is composed of 5160 optical detectors, each containing a photomultiplier tube (PMT) and an onboard digitizer [9]. The PMTs are spread over 86 vertical strings arranged in a hexagonal shape, with a total instrumented volume of approximately $1 \mathrm{~km}^{3}$. The PMTs on a given string are separated 
vertically by $17 \mathrm{~m}$, and the string-to-string separation is roughly $125 \mathrm{~m}$.

At an abstract level, the IceCube detector operates by detecting muons as they travel through the instrumented volume of ice. As the muon travels through the detector, it radiates light [4], which is observed by the PMTs and quantized into discrete hits [10]. The detector uses several trigger criteria. The most commonly used trigger selects time intervals where eight PMTs (with local coincidences) are fired within $5 \mu \mathrm{s}$. When a trigger occurs, all data within a $10-\mu$ s trigger window is saved, becoming an event. If the number of hits in an event is sufficiently large, the muon track reconstruction algorithm is triggered.

There are several challenges for the reconstruction algorithms used in the detector. Varying optical properties of the ice affect reconstruction accuracy, the data may contain outlier hits due to uncorrelated noise, and there are finite computational resources available to tracking code run on-site.

Modeling difficulties: The details of the ice's optical properties are nontrivial to model. Light propagating through the ice is affected by scattering and absorption. These effects cannot be analytically calculated, and the optical properties of the ice vary with depth [5]. In addition, the Cherenkov light originates both directly from the muon and from particle showers initiated by stochastic energy losses of the muon.

Noise: The noise inherent in the data is another challenge. Noise hits can arise, either from the thermal background of the photocathode or from photons generated by radioactive decay inside the PMT [9].

Computational constraints: The reconstruction algorithms are also limited in complexity by the computing resources available at the South Pole. The track reconstruction algorithm has to process about 3000 muons per second, so algorithms with excessive computational demands are discouraged.

\section{Reconstruction improvement}

As shown in the following, augmenting the reconstruction algorithm with some basic filters and classical data analysis techniques results in significant improvement in the reconstruction algorithm's accuracy.

\subsection{Prior IceCube software}

The muon track reconstruction process (outlined in Fig. 2) starts when the number of detected hits exceeds a preset threshold and initiates data collection. After the initial data are collected, the event then passes through a series of basic filters to remove obvious outliers [11].

This is followed by a basic reconstruction algorithm, linefit [12], that disregards the Cherenkov cone and, instead, finds the track that minimizes the sum of the squares of the distances between the track and the hits. More formally, assume that there are $N$ hits; denote the position and time of the $i$ th hit as $\vec{x}_{i}$ and $t_{i}$, respectively. Let the reconstructed muon track have a velocity of $\vec{v}$, and let the reconstructed track pass through point $\vec{x}_{0}$ at time $t_{0}$. Then, linefit reconstruction solves the least-squares optimization problem:

$\min _{t_{0}, \vec{x}_{0}, \vec{v}} \sum_{i=1}^{N} \rho_{i}\left(t_{0}, \vec{x}_{0}, \vec{v}\right)^{2}$

where

$\rho_{i}\left(t_{0}, \vec{x}_{0}, \vec{v}\right)=\left\|\vec{v}\left(t_{i}-t_{0}\right)+\vec{x}_{0}-\vec{x}_{i}\right\|_{2}$

Linefit is an approximation primarily used to generate an initial track or seed for a more sophisticated reconstruction.

The reconstruction algorithm for the sophisticated reconstruction is Single-Photo-Electron-Fit (SPE fit) [3]. SPE fit uses the leastsquares reconstruction, the event data, and a parameterized probability distribution function of scattering in ice [3] to reconstruct the muon track. The SPE fit is the primary reconstruction algorithm used in the initial data selection and filtering run at the detector site, and the fit serves as a seed track to the more complex reconstructions used in off-site data analyses.

\subsection{Algorithm improvement}

If angular deviations of the initial seed are large $\left(>5-10^{\circ}\right)$, the simple subsequent reconstruction, SPE, often does not converge to the global minimum, and the efficiency is degraded. This can be resolved by more advanced but time-consuming reconstruction algorithms or by improving the initial seed, as described here.

As indicated in Eq. (1), a least-squares fit models the muon as a single point moving in a straight line, and hits are penalized quadratically in their distance from this line. Thus, there is an implicit assumption in this model, i.e., all the hits will be near the muon. This assumption has several pitfalls:

1. It does not account for the distinct Cherenkov emission profile from the muon.

2. It ignores the scattering effects of the ice medium. Some of the photons can scatter for over a microsecond, which means that when they are recorded by a PMT, the muon will have traveled over $300 \mathrm{~m}$ away.

3. While the noise reduction steps remove most of the outlier noise, the noise hits that survive can be far from the muon. Because these outliers are given a quadratic weight, they exert a huge influence over the model.

The first two pitfalls occur because the model is incomplete and does not accurately model the data, and the third demonstrates that the model is not robust to noise. The solution to this is twofold: improve the model and increase the noise robustness by replacing least squares with robust statistical techniques.

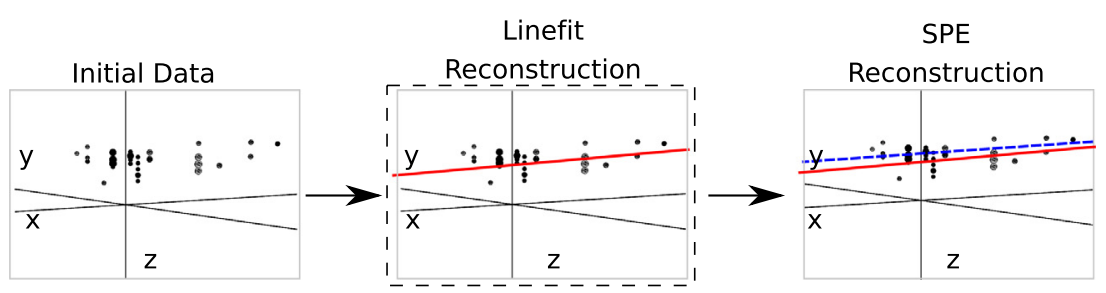

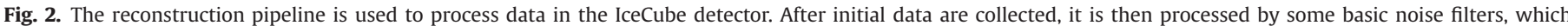

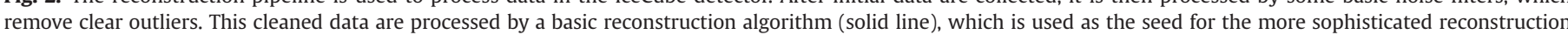

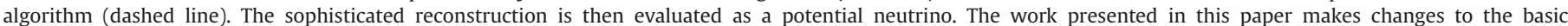
reconstruction step (indicated by the dashed box). 


\subsubsection{Improving the model}

While disregarding the Cherenkov profile is inherent to the simplified model chosen for speed reasons, removing hits generated by photons that scattered for a significant length of time will mitigate the effect of ignoring the photon scattering in the ice. We found that a basic filter could identify these scattered hits, and improve accuracy by almost a factor of two by removing them from the dataset.

More formally, for each hit $h_{i}$, the algorithm looks at all neighboring hits within a neighborhood of $r$, and if there exists a neighboring hit $h_{j}$ with a time stamp that is $t$ earlier than $h_{i}$, then $h_{i}$ is considered a scattered hit, and is not used in the basic reconstruction algorithm. Optimal values of $r$ and $t$ were found to be $156 \mathrm{~m}$ and $778 \mathrm{~ns}$ by tuning them on simulated muon data with an $E^{-2}$ power law spectrum.

\subsubsection{Adding robustness to noise}

As described in Eq. (1), the least squares model gives outliers quadratic weight, whereas we would prefer that outliers had zero weight. There are robust models in classical statistics designed to marginalize outliers. We determined that replacing the leastsquares model with a Huber fit [13] improves the reconstruction accuracy.

More formally, we replace Eq. (1) with the optimization problem:

$\min _{t_{0}, \vec{x}_{0}, \vec{v}} \sum_{i=1}^{N} \phi\left(\rho_{i}\left(t_{0}, \vec{x}_{0}, \vec{v}\right)\right)$

where the Huber penalty function $\phi(\rho)$ is defined as

$\phi(\rho) \equiv\left\{\begin{array}{ll}\rho^{2} & \text { if } \rho<\mu \\ \mu(2 \rho-\mu) & \text { if } \rho \geq \mu\end{array}\right.$.

Here, $\rho_{i}\left(t_{0}, \vec{x}, \vec{v}\right)$ is defined in Eq. (2) and $\mu$ is a constant calibrated to the data (on simulated muon events with an $E^{-2}$ power law spectrum, the optimal value of $\mu$ is $153 \mathrm{~m}$ ).

The Huber penalty function has two regimes. In the near-hit regime $(\rho<\mu)$, hits are assumed to be strongly correlated with the

\section{Table 1}

Median angular resolution (degrees) for reconstruction improvements. The first line is the accuracy of the prior least-squares model, and the subsequent lines are the accuracy measurements from cumulatively adding improvements into the basic reconstruction algorithm.

\begin{tabular}{lc}
\hline Algorithm & $\theta_{\text {med }}$ \\
\hline Linefit reconstruction (least-squares) & 9.917 \\
With addition of logical filter & 5.205 \\
With addition of Huber regression & 4.672 \\
With addition of outlier removal & 4.211 \\
\hline
\end{tabular}

muon's track, and the Huber penalty function behaves like least squares, giving these hits quadratic weight. In the far-hit regime $(\rho \geq \mu)$, hits are given linear weights, as they are more likely to be noise.

In addition to its attractive robustness properties, the Huber fit's weight assignment also has the added benefit that it inherently labels points as outliers (those with $\rho \geq \mu$ ). Thus, once the Huber fit is computed, we can go one step further and simply remove the labeled outliers from the dataset. A better fit is then obtained by computing the least-squares fit on the data with the outliers removed. The entire algorithm has a mean runtime that is approximately six times longer than Linefit's mean runtime.

\subsection{Results}

The goal is to improve the accuracy of the reconstruction in order to better separate neutrinos from cosmic rays. Thus, we present three measurements: (1) the accuracy change between linefit and the new algorithm; (2) the accuracy change when SPE is seeded with the new algorithm instead of linefit; and (3) the improvement in separation between neutrinos and cosmic rays.

To measure the improvement generated by the changes, we use the metric of median angular resolution $\theta_{\text {med. The angular resolu- }}$ tion of a reconstruction is the arc-distance between the reconstruction and the true track. The dataset is drawn from simulated neutrino data and is designed to be similar to that observed by the detector.

We can improve the median angular resolution of the basic reconstruction by $57.6 \%$, as shown in Table 1 . Seeding SPE with the improved basic reconstruction generates an improvement in the angular resolution of $12.9 \%$. These improvements in the reconstruction algorithm result in $10 \%$ fewer atmospheric muons erroneously reconstructed as up-going, and $1 \%$ more muons correctly reconstructed as up-going.

\section{Coincident event improvements}

In the second study, we look at the problem of determining when more than one muon has entered the detector. In the most common case, a single muon will pass though the detector and generate an event before exiting. These events are processed by the pipeline described in Fig. 2. However, for roughly $9 \%$ of the events collected by the data collection algorithm, more than one muon will be passing though the detector simultaneously, an occurrence known as a coincident event.

One of the primary sources of background noise in IceCube analyses is coincident background muons that have been erroneously reconstructed as neutrinos. To see why this occurs, consider the coincident event shown in Fig. 3. There are two clear groups of hits; however, the reconstruction algorithm treats them

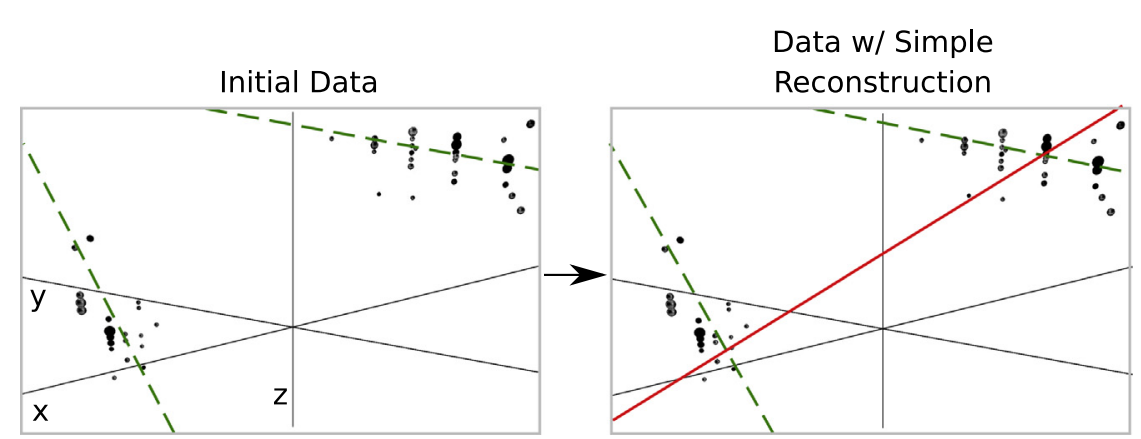

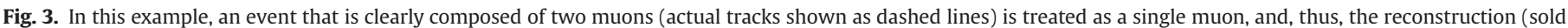
line) is inaccurate. 


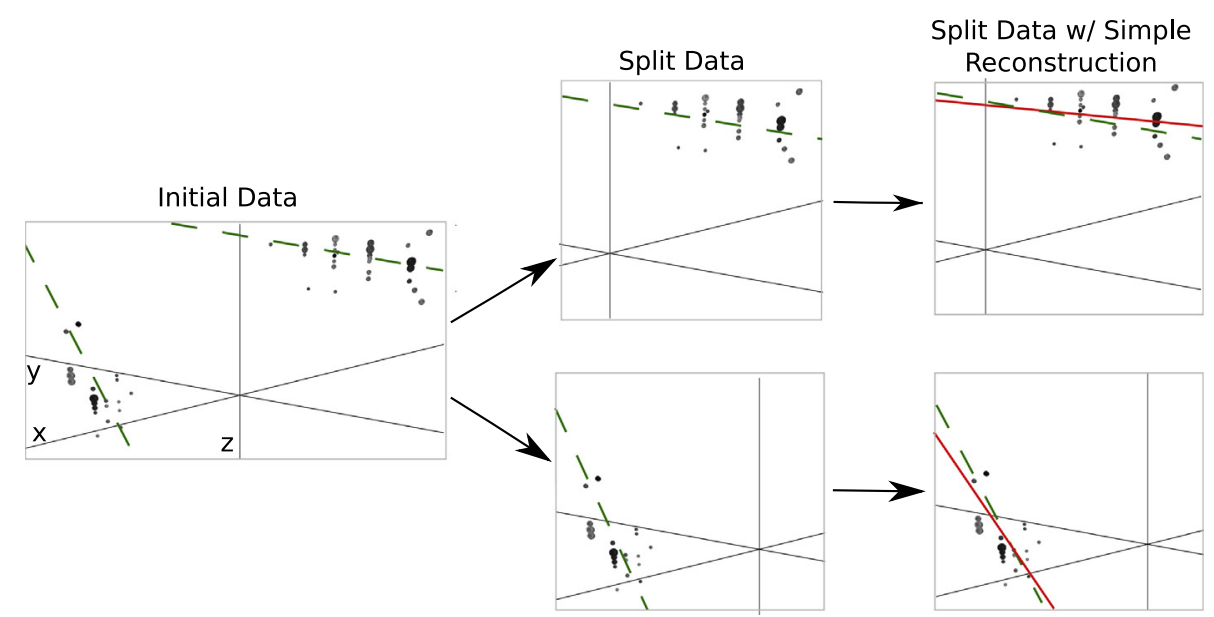

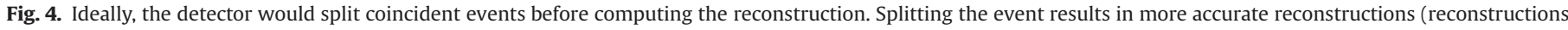
shown as solid lines, true muon tracks shown as dashed lines). Note the difference in the reconstructions compared with Fig. 3.

as a single group, resulting in an erroneous reconstruction. In the ideal case, the reconstruction algorithm would identify coincident events and split them, as in Fig. 4.

The challenge in this example is determining the number of muons in an event. Our studies show that a simple spatial clustering algorithm can solve this classification problem with less than $2 \%$ error.

\subsection{Prior IceCube software}

Coincident events have been a concern in the IceCube analysis [14] for years, and some software has been developed to handle coincident events. As a baseline of comparison, we use the TTrigger software, which is described in Ref. [15].

\subsection{Algorithm improvement}

Here, we present a proximal clustering algorithm. The intuition in proximal clustering is that points local in space and time are probably from the same muon. The proximal clustering algorithm iterates through each pair of hits $(i, j)$ and builds an adjacency matrix $\mathbf{A}$ as

$\mathbf{A}_{i j}= \begin{cases}1 & \text { if }\left\|\Delta x^{2}+\Delta y^{2}+\Delta z^{2}+(c \Delta t)^{2}\right\|_{2} \leq \alpha \\ 0 & \text { otherwise }\end{cases}$

where $\Delta x, \Delta y, \Delta z$, and $\Delta t$ are the space and time differences between the pair of hits, and $\alpha$ is tuned to the data (in this application, the optimal value of $\alpha$ is $450 \mathrm{~m}$ ). The clustering can be recovered by extracting the connected components of the graph defined by A. A connected component of a graph is a subgraph such, that there exists a path between any two vertices of this subgraph.

\subsubsection{Improving the model}

When implemented naively, proximal clustering succeeded for the majority of the events, but it failed if there was a gap in the muon track, which can occur when the muon travels through dusty ice layers with short scattering length. If there is a significantly large gap, the algorithm erroneously separates the hits into two clusters.

To compensate, an additional heuristic is added, track connecting. After the data segmentation is finished, track connecting determines if separate clusters should be combined. It computes the mean position and time of each cluster, and connects a hypothetical muon track $T$ between each pair of subspaces.
It checks if the speed $s$ of the hypothetical track is within $25 \%$ of the speed of light $c$, and it checks that the mean distance between hits and $T$ in both clusters is less than $60 \mathrm{~m}$. If $T$ passes both checks, the clusters are combined.

\subsubsection{Adding robustness to noise}

Proximal clustering is susceptible to noise. Noise hits close to two disjoint tracks will be considered adjacent to both tracks and, thus, can connect the two tracks in the adjacency matrix.

One heuristic that worked well at mitigating this problem was to not use all of the hits in building the adjacency matrix. During data collection, some hits are flagged as having a local coincidence condition, which indicates that both the hits and a neighboring PMT reported a hit. These hits have a high probability of not being noise hits and, thus, exclusively using them to build the adjacency matrix mitigates the problem of erroneously connecting two tracks.

After the proximal clustering algorithm has extracted the tracks from the adjacency matrix, the hits not used in the construction of the adjacency matrix are simply assigned to the closest reconstructed track.

\subsection{Results}

There were two competing goals for coincident event detection algorithms: the algorithm should be conservative enough that events containing single tracks are not erroneously split, and aggressive enough that a useful fraction of coincident events are split correctly. Our algorithm is tuned to keep almost all of the single events correctly unsplit, while still correctly splitting $80 \%$ of the coincident events.

\subsubsection{Measurements}

We modified the reconstruction pipeline shown in Fig. 2, in between the noise cleaning and the basic reconstruction, by adding a step for coincident event detection, as shown in Fig. 4. This step takes cleaned data and attempts to classify the event as a single-track or multiple-track event.

We ran each algorithm on two datasets of simulated data. One dataset comprised single-muon events, and the other dataset comprised multiple-muon events. In each dataset, we measured the classification error $E$, which is the fraction of events that were misclassified. To get a global measurement, we computed the total error $E_{\text {tot }}$, defined as

$E_{\text {tot }}=w_{\text {Single }} E_{\text {Single }}+\alpha w_{\text {Multiple }} E_{\text {Multiple }}$. 
Table 2

Error rates for classification algorithms.

\begin{tabular}{lccc}
\hline Algorithm & $E_{\text {Single }}(\%)$ & $E_{\text {Multiple }}(\%)$ & $E_{\text {tot }}(\%)$ \\
\hline Trivial & 0.0 & 100.0 & 41.5 \\
TTrigger & 11.5 & 31.8 & 23.7 \\
Proximal clustering & 0.2 & 18.9 & 8.0 \\
\hline
\end{tabular}

For computing $E_{\text {tot }}$, we use $w_{\text {Single }}=0.917$ and $w_{\text {Multiple }}=0.083$, which is the frequency in which single-muon and multiple-muon events appear in data simulating the distribution of events that trigger the reconstruction algorithm. We also include a factor of $\alpha$ in the weighting of the multiple-muon events. This factor expresses that mischaracterizing a multiple-muon event as a single-muon event degrades the quality of most higher-order analysis more than the reverse mischaracterization. In our calculations, we use a value of $\alpha=5$.

We present the results for the coincident event problem by measuring how well each algorithm performs at determining the number of subspaces in an event.

There are two natural comparisons for the work: the prior software TTrigger, as well as the trivial algorithm, which always classifies each event as a single-track event. Clearly, the latter will always get the single-track events correct, and always get the multiple-track events wrong. We provide a comparison of these techniques in Table 2. As shown, the new algorithm classifies the number of muons in the detector $66 \%$ better than TTrigger.

\section{Conclusions}

We found that significant improvements can be achieved in the IceCube's online track reconstruction by employing some classical data analysis algorithms. Optimizing data filtering and refining the least-square model have led to significant improvements in the accuracy of the reconstruction direction. The new reconstruction software is fast enough to run on-site, and is now included in all IceCube analyses.

We also looked at the problem of determining the number of muons in the detector. We found that proximal clustering with some basic heuristics could correctly determine whether an event contained a single muon or multiple muons with less than $2 \%$ error, yielding a $66 \%$ improvement over the prior software.

\section{References}

[1] IceCube Collaboration, IceCube Webpage, 〈http://icecube.wisc.edu/ $\rangle$.

[2] A. Achterberg, et al., Astropart. Phys. 26 (3) (2006) 155.

[3] J. Ahrens, et al., Nucl. Instrum. Methods Phys. Res. Sec. A 524 (2004) 169.

[4] M.G. Aartsen, et al., Nucl. Instrum. Methods Phys. Res. Sec. A (2013) 73.

[5] M. Ackermann, et al., J. Geophys. Res. 111 (D13) (2006) D13203.

[6] F. Hirsch, ATLAS Collaboration, Nucl. Instrum. Methods Phys. Res. Sec. A: Acceler., Spectrom., Detect. Assoc. Equip. 650 (1) (2011) 218.

[7] R.S. Chivukulaa, M. Goldena, E.H. Simmons, Nucl. Phys. B 363 (1) (1991) 83.

[8] S. Ellis, J. Huston, K. Hatakeyama, P. Loch, M. Tönnesmann, Prog. Part. Nucl. Phys. (60) (2008) 484.

[9] R. Abbasi, et al., Nucl. Instrum. Methods Phys. Res. Sec. A 618 (2010) 139.

[10] R. Abbasi, et al., Nucl. Instrum. Methods Phys. Res. Sec. A 601 (3) (2009) 294

[11] M. Ackermann, Searches for signals from cosmic point-like sources of high energy neutrinos in 5 years of AMANDA-II data (Ph.D. thesis), HumboldtUniversität zu Berlin, 2006.

[12] V. Stenger, Track fitting for DUMAND-II Octagon Array (Technical Report), University of Hawaii at Manoa, 1990.

[13] S. Boyd, L. Vandenberghe, Convex Optimization, Cambridge University Press, 2009, pp. 298-300.

[14] R. Abbasi, et al., Phys. Rev. D 83 (1) (2011), http://link.aps.org/doi/10.1103/ PhysRevD.83.012001.

[15] D. Chirkin, Measurement of the atmospheric neutrino energy spectrum with IceCube, Proceedings of the 31st ICRC, 2009. 